\title{
Scalar-Wave Localization in a Two-Component Composite
}

\author{
Ping Sheng and Zhao-Qing Zhang (a) \\ Corporate Research Science Laboratories, Exxon Research and Engineering Company, Annandale, New Jersey 08801
}

(Received 23 June 1986)

\begin{abstract}
Scalar-wave localization characteristics in a two-component composite are calculated as a function of frequency, relative volume fraction, and impedance contrast between the components. It is found that the occurrence of localization requires a minimum impedance contrast whose value is defined by a critical point on the localization phase diagram. Furthermore, localization is shown to be closely tied to resonant scattering, and there is a multitude of mobility edges and localization regions associated with resonance harmonics.
\end{abstract}

PACS numbers: $42.20 .-\mathrm{y}, 03.40 . \mathrm{Kf}, 43.20 .+\mathrm{g}, 62.65 .+\mathrm{k}$

As a wave phenomenon in disordered systems, Anderson localization is recognized to be common to both quantum particles and classical waves, i.e., elastic and electromagnetic waves. While up to now most of the works on localization have focused on electronic systems, recently there is an increasing interest in the study of classical-wave localization, ${ }^{1-12}$ since the classical waves offer both the potential for more direct observation of localization as well as the possibility of novel localization characteristics distinct from those of electrons. As a first step towards the clarification of classical-wave-localization behavior in three-dimensional random systems, in this work we use the mathematical framework developed by Vollhardt and Wölfle $^{13}$ to calculate the scalar-wave-localization phase diagram for a two-component composite. Our results indicate that the occurrence of mobility edge requires the impedance contrast between the components composing the medium to exceed a certain minimum value, and the point at which localization first appears represents an isolated critical point on the localization phase diagram (the variables being frequency, impedance contrast, and relative volume fraction). Furthermore, we found the localization to be closely tied to resonant scattering, and there could be a multitude of mobility edges, localization regions, and critical points associated with resonance harmonics.

Consider a composite consisting of two components with indices of refraction $n_{1}$ and $n_{2}\left(>n_{1}\right)$. Impedence contrast of the medium is defined to be $m=n_{2} / n_{1}(>1)$. We will limit our consideration to cases where $n_{1}$ and $n_{2}$ are real, i.e., the wave is locally propagating in nature. Volume fraction of component 1 is denoted by $1-p$ and that of component 2 by $p$. At $p \rightarrow 0$ or $p \rightarrow 1$, our composite microstructure will be assumed to consist of spheres of the minority component, with diameter $d$, randomly dispersed in the matrix of the majority component. In the intermediate range we will assume the microstructure to be that resulting from a symmetric randomization of the two components.

The scalar-wave amplitude $u$ satisfies the wave equa- $\operatorname{tion}^{14}$

$$
\left[1 / c^{2}(\mathbf{r})\right]\left(\partial^{2} u / \partial t^{2}\right)-\nabla^{2} u=0,
$$

where the wave speed $c$ takes the value of $c_{1}$ in component 1 and the value of $c_{2}=c_{1} / m\left(<c_{1}\right)$ in component 2. Since $u$ is assumed to satisfy Eq. (1) for all spatial points $\mathbf{r}$, it is straightforward to show that the amplitude $u$ and its normal derivative must be continuous across an interface. In a random medium, wave propagation can have different characters when viewed on different length scales. At scales less than the mean free path of scattering, $l$, the propagation is basically wavelike. However, at scales much larger than $l$ the numerous scatterings make the energy transfer diffusionlike with a diffusion constant $D_{0}=\bar{c} l / 3$, where $\bar{c}$ is the effective wave speed of the medium. Provided that $\bar{c}$ is known, $D_{0}$ for a wave of frequency $\omega$ can be calculated from the transport theory as ${ }^{7}$

$$
D_{0}=-\frac{\bar{c}}{24 \pi^{5} N}\left[\int_{0}^{\pi} d \theta \sin \theta(\cos \theta-1) \sigma(\theta)\right]^{-1},
$$

where

$$
\sigma(\theta)=\left(1 / 4 \pi^{4}\right)\left[(1-p)\left|f_{1}(\theta)\right|^{2}+p\left|f_{2}(\theta)\right|^{2}\right] ;
$$

$N=\left[(\pi / 6) d^{3}\right]^{-1}$ is the number of scatterers per unit volume, $f_{1(2)}(\theta)$ is the scattering amplitude of a plane wave of frequency $\omega$ propagating in a medium of speed $\bar{c}$ impinging on a sphere of component 1 (2) and diameter $d$, and $\theta$ is the scattering angle defined relative to the incident direction. The scattering amplitude $f_{1(2)}(\theta)$ can be readily obtained from the solution of a simple boundary-value problem. ${ }^{15}$

To calculate $\bar{c}$, we start with a microgeometry appropriate to $p \simeq 0$ or $p \simeq 1$ : spherical scatterers well dispersed in a matrix medium. Let us suppose for the moment that the matrix is component 1 and the scatterers component 2 (suitable for $p \simeq 0$ ). The Green's function of the wave equation (1) in the frequency-momentum variables $(\omega, k)$ is given by

$$
G(k, \omega)=\left[\left(\omega / c_{1}\right)^{2}-k^{2}-\Sigma\right]^{-1},
$$


where the self-energy $\Sigma=\Sigma_{R}+i \Sigma_{I}$ is directly related to the forward-scattering amplitude $f(0)$ calculated from the scattering of a plane wave in medium 1 by a sphere of component 2 :

$$
\Sigma=-24 p d^{-3} f(0) \text {. }
$$

In Eq. (5) the factor $p$ is present to account for the volume fraction of scatterers. From Eq. (4) it is clear that the medium speed $c_{1}$ should be renormalized to a new value

$$
\bar{c}_{1}^{-1}=\operatorname{Re}\left[c_{1}^{-2}-\left(\Sigma / \omega^{2}\right)\right]^{1 / 2}
$$

$\left\{\operatorname{Im}\left[c_{1}^{-2}-\left(\Sigma / \omega^{2}\right)\right]^{1 / 2}\right.$ represents effective attenuation due to scattering loss $\}$. Our numerical evaluation of $\bar{c}_{1}$ has shown that at low frequencies where the wavelength is much larger than the scale of the inhomogeneities, $\bar{c}_{1}$ is almost identical to the effective medium value $c_{e}$ calculated from the condition of vanishing average forward-scattering amplitude (for a wave traveling in a medium composed of two symmetrically randomized components). This agreement with $c_{e}$ persists to practically all values of $p$. If we exchange the roles of component 1 and component 2 , a renormalized $\bar{c}_{2}$ can be similarly defined, and at low frequencies $\bar{c}_{2} \simeq c_{e} \simeq \bar{c}_{1}$ for all values of $p$. As frequency increases, $\bar{c}_{1}$ and $\bar{c}_{2}$ deviate from $c_{e}$ and the validity of $\bar{c}_{1}$ (2) becomes limited to composition regions of $p \simeq 0$ and $p \simeq 1$ where a matrix phase is well defined and the application of perturbation theory is justifiable. ${ }^{16}$ To interpolate between these two compositional limits, we observe that for a high-frequency pulse whose width is $<<d$ and which can therefore resolve its immediate environment as either component 1 or component 2 , the effective pulse speed is given by the geometric-optics value $\left[(1-p) / c_{1}+p /\right.$ $\left.c_{2}\right]^{-1}$. By replacing $c_{1}$ and $c_{2}$ in this expression by $\bar{c}_{1}$ and $\bar{c}_{2}$, we obtain an interpolation formula

$$
1 / \bar{c}=(1-p) / \bar{c}_{1}+p / \bar{c}_{2}
$$

for $\bar{c}$, which tends to the correct frequency limits as well as the correct compositional limits. This value of $\bar{c}$ will be used in the calculation of the diffusion constant $D_{0}$, Eq. (2), and $f_{1(2)}(\theta)$.

Localization of the wave occurs when the effective diffusion constant approaches zero. According to the scaling theory of localization, ${ }^{17}$ the way this happens is as follows. Near the mobility edge, properties of the wave field are homogeneous only on a scale greater than a correlation length $\xi>l$. At scales less than $\xi$, diffusion constant varies monotonically from $D_{0}$ at the scale of the mean free path $l$ to $D_{0} l / \xi$ at the scale of $\xi$. (This is analogous to diffusion on a fractal structure which has a lower length scale $l$ and an upper length scale $^{18} \xi$.) Far away from the mobility edge $\xi \simeq l$, and the effective diffusion constant is given by $D_{0}$. However, as one approaches the mobility edge $\xi$ diverges, and the effective diffusion constant vanishes as a consequence. Vollhardt and Wölfe ${ }^{13}$ have identified mathematically the class of maximally crossed diagrams as the dominant multiple-scattering contribution to the renormalization of the diffusion constant near the mobility edge. The resulting correction to $D_{0}$ is given by ${ }^{7,13}$

$$
D(\omega)=D_{0}(\omega) \frac{l}{\xi}=D_{0}(\omega)\left[1-\frac{3 \gamma(\omega)}{\pi}\left(\frac{\bar{c}}{\omega}\right)^{3} q(\omega)\right],
$$

where $q(\omega)=K / l(\omega)$ is a cutoff wave vector, $K$ is a constant, and $\gamma=-24 d^{3} \operatorname{Im}\left[(1-p) f_{1}(0)+p f_{2}(0)\right]$ is related to the total scattering cross section. We observe that $q$ is bounded by a maximum value $q_{\max }$ $=2 \pi / d$, since $d$ is the minimum scale length of the medium. That means if there is a minimum value of $l$ given by $l_{\min }$, then the maximum value of $K$ is given by $K_{\max }=2 \pi l_{\min } / d$. Since $l_{\min } \sim d$, we have $K_{\max }$ $=2 \pi$. In the following calculation we will use $q=2 \pi /$ $l(\omega)$ as the cutoff wave vector. While the use of a particular value of $K$ will not alter any qualitative features of our result, the quantitative implications of picking $K_{\max }$ will be noted and contrasted with those of using a smaller $K$. In this context it should be mentioned that the diffusion constants calculated by use of $K=1$ and $2 \pi$, for parameters appropriate to Genack's optical-transmission experiment using polystyrene spheres, ${ }^{11}$ are both in reasonable accord with the measured value(s). For this particular case the value of $K$ does not have a significant influence on the calculated diffusion constant, which is on the order of $(1.1-1.5) \times 10^{6} \mathrm{~cm}^{2} / \mathrm{sec}$ for $p$ varying from 0.4 to 0.6 , $m=1.59, d=0.5-0.8 \mu \mathrm{m}, \quad$ and wavelength in air $=5896 \AA$.

In Fig. 1 the mobility-edge contours calculated from the condition $D(\omega)=0$ are plotted as a function of $p$ and $\omega d / c_{1}$. Each contour, which represents a single value of the impedance contrast $m$, separates the localized region inside the contour from the delocalized region outside. As seen in the figure, for impedance contrast of up to $m=3$ there are three large regions of localization centered roughly at $\omega d / c_{1} \simeq 1.7,2.9$, and 4. As a function of increasing $m$, localization first appears in the middle region in the form of an isolated critical point with $m=2.11, p=0.35$, and $\omega d / c_{1}$ $=3.1$. The use of $K_{\max }$ in the calculation means $m=2.11$ is the minimum impedance contrast required for scalar-wave localization in our present model. As the middle region grows in size, the region centered at $\omega d / c_{1} \simeq 4$ starts to nucleate from a second critical point at $m \simeq 2.12, p \simeq 0.44$, and $\omega d / c_{1} \simeq 4.15$. This is followed by the nucleation and growth of the $\omega d / c_{1}=1.7$ region when $m$ increases beyond 2.25 . We have also examined the case of letting $K=1$. It was found that the location and order of appearance of the localization regions still remain the same, except 


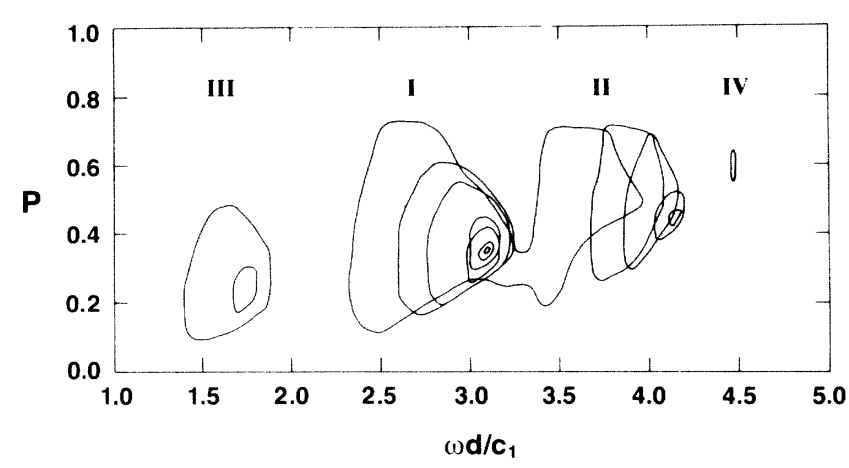

FIG. 1. Mobility-edge contours projected on the $p-\omega d / c_{1}$ plane. The order of appearance of the three regions as $m$ is increased is noted by I, II, III, and IV. In each region the smaller contours always appear before the larger ones. In I, the first six contours correspond to $m=2.107,2.11,2.12$, $2.14,2.22$, and 2.3. The seventh one, $m=2.50$, extends over to region II. In II the first four contours correspond to $m=2.12,2.14,2.22$, and 2.3. In III the two contours correspond to $m=2.3$ and 2.5 . In IV the narrow strip of localized region corresponds to $m=2.5$. The value of $K$ used in the calculation is $2 \pi$.

now the minimum impedance contrast is raised to $m=2.48$.

Examination of the scattering cross sections in the three regions of localization shows that they are directly related to the $i=1,2,3$ resonances of the component- 2 spheres, where $i$ denotes the angular degeneracy of the resonant mode langular variation of the resonant mode is given by $P_{i}(\cos \theta)$, where $P$ is the Legendre polynomial]. The localized region centered at $\omega d / c_{1} \simeq 2.9$ is predominantly associated with the $i=2$ resonant scattering. The reason why this region appears first has to do with the fact that there is an optimal frequency range, centered about $\omega d / c_{1} \simeq 2.5$, for the occurrence of localization. If the frequency is too low, scattering is weak and therefore $\gamma(\omega)$ is small. On the other hand, at high frequencies the decrease of wavelength inevitably limits the growth of $\xi$ and thereby also inhibits localization. This leaves the intermediate-frequency range, which has a wavelength in the fast medium (component 1 in our case) 1.5-3 times that of $d$, the most susceptible to localization. In this frequency range localization first occurs when the enhancement of the scattering cross section by a particular resonance is just enough to bring $D(\omega)$ to zero ( $i=2$ resonance in our case). As the impedance contrast becomes larger, the resonance enhancement increases, and this broadens the localization region. It should be noted that resonances affect not only the scattering cross section but also the effective speed $\bar{c}$, making its value an oscillating function of frequency. Also, we have found that the scattering of a wave in a fast medium by a scatterer of the slower component is

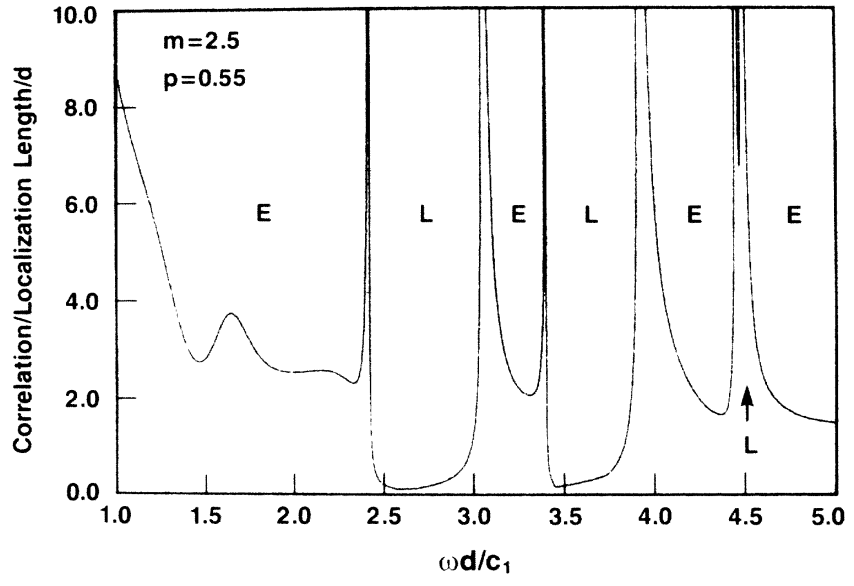

FIG. 2. Correlation length and localization length plotted as a function of the frequency variable $\omega d / c_{1}$. E denotes extended region. The curves in extended regions represent the correlation length $\xi$. L denotes localized region. The curves in localized regions represent the localization length $\lambda$. Values of $m$ and $p$ are given in the figure.

much stronger than if the medium and the scatterer components were switched around. In our case, this accounts for the nonsymmetric shapes of the localization regions about $p=0.5$, since for $p<0.5$ we have predominantly component- 2 scatterers.

In localized regimes, the localization length $\lambda$ is the counterpart to the length $\xi$ in extended regimes. An extension of the Vollhardt and Wölfle theory ${ }^{19}$ gives the following equation for $\lambda$ :

$$
1=\frac{3 \gamma(\omega)}{\pi}\left(\frac{\bar{c}}{\omega}\right)^{3} \int_{0}^{q(\omega)} \frac{k^{2}}{k^{2}+\lambda^{-2}} d k
$$

In Fig. 2 the localization length as calculated from Eq. (8), and the correlation length as calculated from Eq. (7), are plotted versus $\omega d / c_{1}$. The values of $p$ and $m$ are given in the figure. Three points about Fig. 2 should be noted. First, at low frequencies we have $\xi \simeq l$, so the increase in $\xi$ reflects the increase of the mean free path $l$. Also, the undulation seen in the low-frequency region is the manifestation of a scatterer resonance. Second, the divergences of $\xi$ and $\lambda$ at the mobility edges are verified to have the form $\left(\omega-\omega^{*}\right)^{-1}$, where $\omega^{*}$ denotes the location of a mobility edge. Third, due to the uncertainty in the value of $K$, the values of $\xi$ and $\lambda$ should only be viewed as an order-of-magnitude estimate. A smaller $K$ would shift the mobility edges and increase the minimum values of $\lambda$ (in fact, by requiring $\lambda>d$ we can limit the maximum value of $K$ to $\leq \pi / 2)$; however, the qualitative behavior would still remain the same. An interesting question is whether the divergence exponent for $\xi$ would remain 1 at the isolated critical point where the 
localization region shrinks down to a point. By calculating the coordinate of the isolated critical point $\left(\omega^{* *}, p^{* *}, m^{* *}\right)$ to a high degree of accuracy, we have determined that $\xi \sim\left(\omega-\omega^{* *}\right)^{-2}$, i.e., the exponent at the isolated critical point is twice that of the usual critical exponent.

In conclusion, our calculation indicates that localization of the scalar wave should occur in a twocomponent composite when the impedance contrast exceeds a certain minimum value that is in the range of 2.1-2.5. Moreover, the optimal parameter range for the observation of localization is found to be $p \sim 0.35-0.45$ and $\omega d / c_{1} \sim 2-3$. Based on the empirical observation that the scalar wave reproduces much of the classical-wave phenomena despite the vector character of the classical waves, we expect our results to be qualitatively generalizable to elastic and electromagnetic waves. However, a full quantitative account of the classical-wave localization remains a challenge to be addressed by further theoretical and experimental studies.

We wish to thank A. Genack for numerous discussions of his experimental results prior to publication.

(a) On leave from Department of Physics and Astronomy, San Francisco State University, San Francisco, CA 94132, and Institute of Physics, Academia Sinica, Beijing, People's Republic of China.

1S. John, Phys. Rev. Lett. 53, 2169 (1984).

2P. W. Anderson, Philos. Mag. B 52, 505 (1985).

${ }^{3}$ M. Y. Azbel, Phys. Rev. B 28, 4106 (1983).

${ }^{4}$ S. M. Cohen and J. Machta, Phys. Rev. Lett. 54, 2242 (1985).

${ }^{5}$ M. P. Van Albada and A. Lagendijk, Phys. Rev. Lett. 55, 2692 (1985).

6P. E. Wolf and G. Maret, Phys. Rev. Lett. 55, 2696 (1985).
7T. R. Kirkpatrick, Phys. Rev. B 31, 5746 (1985).

${ }^{8}$ L. Tsang and A. Ishimaru, J. Opt. Soc. Am. A 1, 836 (1984).

${ }^{9}$ M. J. Stephen, Phys. Rev. Lett. 56, 1809 (1986).

${ }^{10}$ K. Arya, Z. B. Su, and J. L. Birman, Phys. Rev. Lett. 54, 1559 (1985).

${ }^{11} \mathrm{~A} . \mathrm{Z}$. Genack, to be published.

12P. Sheng, B. White, Z. Q. Zhang, and G. Papanicolaou, Phys. Rev. B 34, 4757 (1986).

${ }^{13}$ D. Vollhardt and P. Wölfle, Phys. Rev. Lett. 45, 842 (1980), and Phys. Rev. B 22, 4666 (1980), and Phys. Rev. Lett. 48, 669 (1982). It should be mentioned that the theory of Vollhardt and Wölfle has been used to calculate the mobility edge and localization length of the tight-binding electron models [A. D. Zdetsis et al., Phys. Rev. B 32, 7811 (1985)]. The results are in excellent agreement with numerical-simulation data.

${ }^{14} \mathrm{An}$ alternative form for the scalar-wave equation is $\partial^{2} u / \partial t^{2}-\nabla \cdot\left[c^{2}(\mathbf{r}) \nabla u\right]=0$. The interfacial boundary condition consistent with this form is the continuity of $u$ and of $c^{2}$ times the normal derivative of $u$. For the acoustic-wave equation, if we let the density $\rho$ be constant and the compressional modulus $\beta(\mathbf{r})$ be spatially varying, then one gets Eq. (1) if $u$ is identified as pressure $P$. On the other hand, if one identifies $u$ as the local volume dilatation ( $u=\nabla \cdot \mathbf{s}$, where $\mathbf{s}$ is the displacement vector), then one gets the alternative form above. Since $P=\beta(\mathbf{r}) \nabla \cdot \mathbf{s}$, solution of one is equivalent to the solution of the other.

${ }^{15}$ See, for example, J. D. Jackson, Classical Electrodynamics (Wiley, New York, 1975), 2nd ed., p. 391.

${ }^{16}$ In fact, as $\omega$ increases $\bar{c}_{1}$ and $\bar{c}_{2}$ approach the limits of $p=0$ and $p=1$, respectively. That is, $\bar{c}_{1} \rightarrow c_{1}$ and $\bar{c}_{2} \rightarrow c_{2}$ as $\omega \rightarrow \infty$.

${ }^{17}$ E. Abraham, P. W. Anderson, D. C. Licciardello, and T. V. Ramakrishnan, Phys. Rev. Lett. 42, 673 (1979).

18W. D. Dozier, J. M. Drake, and J. Klafter, Phys. Rev. Lett. 56, 197 (1986).

${ }^{19}$ See, for example, E. N. Economou, Green's Function in Quantum Physics (Springer-Verlag, New York, 1983), 2nd ed., p. 191. 